Int. Journal of Math. Analysis, Vol. 8, 2014, no. 18, 895 - 900

HIKARI Ltd, www.m-hikari.com http://dx.doi.org/10.12988/ijma.2014.4390

\title{
Generalization of Some Inequalities for the Ratio of Gamma Functions
}

\author{
K. Nantomah and M. M. Iddrisu \\ Department of Mathematics \\ University for Development Studies \\ Navrongo Campus, P. O. Box 24 \\ Navrongo, UE/R, Ghana \\ E. Prempeh \\ Department of Mathematics \\ Kwame Nkrumah University of Science and Technology \\ Kumasi, Ghana
}

Copyright (c) 2014 K. Nantomah, M. M. Iddrisu and E. Prempeh. This is an open access article distributed under the Creative Commons Attribution License, which permits unrestricted use, distribution, and reproduction in any medium, provided the original work is properly cited.

\begin{abstract}
We present some monotonic functions and some generalized inequalities involving the ratios of analogues of the Gamma function.
\end{abstract}

Mathematics Subject Classification: 33B15, 26A48

Keywords: Gamma Function, $p$-analogue, $q$-analogue, $k$-analogue, Inequality

\section{Introduction}

The classical Euler's Gamma function $\Gamma(t)$ is commonly defined as

$$
\Gamma(t)=\int_{0}^{\infty} e^{-x} x^{t-1} d x, \quad t>0 .
$$


The $p$-digamma function $\psi_{p}(t)$, $q$-digamma function $\psi_{q}(t)$ and $k$-digamma function $\psi_{k}(t)$ are respectively defined as follows.

$$
\psi_{p}(t)=\frac{d}{d t} \ln \left(\Gamma_{p}(t)\right)=\frac{\Gamma_{p}^{\prime}(t)}{\Gamma_{p}(t)}, \quad t>0 .
$$

where $\Gamma_{p}(t)$ is the $p$-analogue of the Gamma function defined by (see [2], [3])

$$
\begin{gathered}
\Gamma_{p}(t)=\frac{p ! p^{t}}{t(t+1) \ldots(t+p)}=\frac{p^{t}}{t\left(1+\frac{t}{1}\right) \ldots\left(1+\frac{t}{p}\right)}, \quad p \in N, \quad t>0 \\
\psi_{q}(t)=\frac{d}{d t} \ln \left(\Gamma_{q}(t)\right)=\frac{\Gamma_{q}^{\prime}(t)}{\Gamma_{q}(t)}, \quad t>0
\end{gathered}
$$

where $\Gamma_{q}(t)$ is the $q$-analogue of the Gamma function defined by (see [4])

$$
\Gamma_{q}(t)=(1-q)^{1-t} \prod_{n=1}^{\infty} \frac{1-q^{n}}{1-q^{t+n}}, \quad q \in(0,1), \quad t>0,
$$

and

$$
\psi_{k}(t)=\frac{d}{d t} \ln \left(\Gamma_{k}(t)\right)=\frac{\Gamma_{k}^{\prime}(t)}{\Gamma_{k}(t)}, \quad t>0
$$

where $\Gamma_{k}(t)$ is the $k$-analogue of the Gamma function defined by (see [1], [5])

$$
\Gamma_{k}(t)=\int_{0}^{\infty} e^{-\frac{x^{k}}{k}} x^{t-1} d x, \quad k>0, \quad t>0 .
$$

In a recent paper [6], Nantomah and Iddrisu proved that the following double inequalities hold:

$$
0<\frac{\Gamma_{k}(\alpha+t)}{\Gamma_{p}(\alpha+t)}<\frac{e^{(t-1)\left(\frac{\ln k-\gamma}{k}\right)} \Gamma_{k}(\alpha+1)}{t p^{t-1} \Gamma_{p}(\alpha+1)}, \quad k>0, p \in N
$$

and

$$
0<\frac{\Gamma_{k}(\alpha+t)}{\Gamma_{q}(\alpha+t)}<\frac{e^{(-t)\left(\frac{\ln k-\gamma}{k}\right)} \Gamma_{k}(\alpha+1)}{t(1-q)^{1-t} \Gamma_{q}(\alpha+1)}, \quad k>0, q \in(0,1)
$$

for $t \in(0,1)$ and for a positive real number $\alpha$.

Our objective in this paper is to establish some generalizations of the inequalities (8) and (9). 


\section{Preliminary Results}

The following auxiliary results are crucial to the main results of the paper.

Lemma 2.1. The functions $\psi_{p}(t), \psi_{q}(t)$ and $\psi_{k}(t)$ as defined above have the following series representations.

$$
\begin{gathered}
\psi_{p}(t)=\ln p-\sum_{n=0}^{p} \frac{1}{n+t}, \quad p \in N, \quad t>0 \\
\psi_{q}(t)=-\ln (1-q)+\ln q \sum_{n=0}^{\infty} \frac{q^{t+n}}{1-q^{t+n}}, \quad q \in(0,1), \quad t>0 \\
\psi_{k}(t)=\frac{\ln k-\gamma}{k}-\frac{1}{t}+\sum_{n=1}^{\infty} \frac{t}{n k(n k+t)}, \quad k>0, \quad t>0 .
\end{gathered}
$$

where $\gamma$ is the Euler-Mascheroni's constant.

Proof. See [3], [5] and [6] and the references therein.

Lemma 2.2. Let $a>0, b>0$ and $t>0$. Then,

$$
-a\left(\frac{\ln k-\gamma}{k}\right)+b \ln p+\frac{a}{t}+a \psi_{k}(t)-b \psi_{p}(t)>0
$$

Proof. Using the series representations in equations (10) and (12) we have,

$$
\begin{aligned}
-a\left(\frac{\ln k-\gamma}{k}\right)+b \ln p+\frac{a}{t}+a \psi_{k}(t) & -b \psi_{p}(t) \\
& =a \sum_{n=1}^{\infty} \frac{t}{n k(n k+t)}+b \sum_{n=0}^{p} \frac{1}{(n+t)}>0 .
\end{aligned}
$$

Lemma 2.3. Let $a>0, b>0$ and $\alpha+\beta t>0$. Then

$$
-a\left(\frac{\ln k-\gamma}{k}\right)+b \ln p+\frac{a}{t}+a \psi_{k}(\alpha+\beta t)-b \psi_{p}(\alpha+\beta t)>0
$$

Proof. This follows directly from Lemma 2.2.

Lemma 2.4. Let $a>0, b>0$ and $t>0$. Then,

$$
-a\left(\frac{\ln k-\gamma}{k}\right)+b \ln (1-q)+\frac{a}{t}+a \psi_{k}(t)-b \psi_{q}(t)>0
$$


Proof. Using the series representations in equations (11) and (12) we have,

$$
\begin{aligned}
-a\left(\frac{\ln k-\gamma}{k}\right)+b \ln (1-q)+ & \frac{a}{t}+a \psi_{k}(t)-b \psi_{q}(t) \\
& =a \sum_{n=1}^{\infty} \frac{t}{n k(n k+t)}-b \ln q \sum_{n=0}^{\infty} \frac{q^{t+n}}{1-q^{t+n}}>0
\end{aligned}
$$

Lemma 2.5. Let $a>0, b>0$ and $\alpha+\beta t>0$. Then,

$$
-a\left(\frac{\ln k-\gamma}{k}\right)+b \ln (1-q)+\frac{a}{t}+a \psi_{k}(\alpha+\beta t)-b \psi_{p}(\alpha+\beta t)>0
$$

Proof. This follows directly from Lemma 2.4.

\section{Main Results}

We now state and prove the results of this paper.

Theorem 3.1. Define a function $\Lambda$ by

$$
\Lambda(t)=\frac{t^{a \beta} e^{-a \beta t\left(\frac{\ln k-\gamma}{k}\right)} \Gamma_{k}(\alpha+\beta t)^{a}}{p^{-b \beta t} \Gamma_{p}(\alpha+\beta t)^{b}}, \quad t \in(0, \infty), k>0, p \in N .
$$

where $a, b, \alpha, \beta$ are positive real numbers. Then $\Lambda$ is increasing on $t \in(0, \infty)$ and the inequality

$$
0<\frac{\Gamma_{k}(\alpha+\beta t)^{a}}{\Gamma_{p}(\alpha+\beta t)^{b}}<\frac{e^{a \beta(t-1)\left(\frac{\ln k-\gamma}{k}\right)} \Gamma_{k}(\alpha+\beta)^{a}}{t^{a \beta} p^{b \beta(t-1)} \Gamma_{p}(\alpha+\beta)^{b}}
$$

holds for every $t \in(0,1)$.

Proof. Let $g(t)=\ln \Lambda(t)$ for every $t \in(0, \infty)$. Then,

$$
\begin{aligned}
g(t) & =\ln \frac{t^{a \beta} e^{-a \beta t\left(\frac{\ln k-\gamma}{k}\right)} \Gamma_{k}(\alpha+\beta t)^{a}}{p^{-b \beta t} \Gamma_{p}(\alpha+\beta t)^{b}} \\
& =-a \beta t\left(\frac{\ln k-\gamma}{k}\right)+b \beta t \ln p+a \beta \ln t+a \ln \Gamma_{k}(\alpha+\beta t)-b \ln \Gamma_{p}(\alpha+\beta t)
\end{aligned}
$$

Then,

$$
\begin{aligned}
g^{\prime}(t) & =-a \beta\left(\frac{\ln k-\gamma}{k}\right)+b \beta \ln p+\frac{a \beta}{t}+a \beta \psi_{k}(\alpha+\beta t)-b \beta \psi_{p}(\alpha+\beta t) \\
& =\beta\left[-a\left(\frac{\ln k-\gamma}{k}\right)+b \ln p+\frac{a}{t}+a \psi_{k}(\alpha+\beta t)-b \psi_{p}(\alpha+\beta t)\right]>0
\end{aligned}
$$


as a result of Lemma 2.3. This proves that $g$ is increasing on $t \in(0, \infty)$. Hence $\Lambda$ is increasing on $t \in(0, \infty)$. Thus, for every $t \in(0,1)$ we have

$$
\Lambda(0)<\Lambda(t)<\Lambda(1)
$$

yielding

$$
0<\frac{\Gamma_{k}(\alpha+\beta t)^{a}}{\Gamma_{p}(\alpha+\beta t)^{b}}<\frac{e^{a \beta(t-1)\left(\frac{\ln k-\gamma}{k}\right)} \Gamma_{k}(\alpha+\beta)^{a}}{t^{a \beta} p^{b \beta(t-1)} \Gamma_{p}(\alpha+\beta)^{b}} .
$$

Corollary 3.2. If $t \in[1, \infty)$, then the following inequality holds.

$$
\frac{e^{a \beta(t-1)\left(\frac{\ln k-\gamma}{k}\right)} \Gamma_{k}(\alpha+\beta)^{a}}{t^{a \beta} p^{b \beta(t-1)} \Gamma_{p}(\alpha+\beta)^{b}} \leq \frac{\Gamma_{k}(\alpha+\beta t)^{a}}{\Gamma_{p}(\alpha+\beta t)^{b}}
$$

Proof. If $t \in[1, \infty)$, then we have $\Lambda(1) \leq \Lambda(t)$ yielding the result.

Theorem 3.3. Define a function $\Upsilon$ by

$$
\Upsilon(t)=\frac{t^{a \beta} e^{-a \beta t\left(\frac{\ln k-\gamma}{k}\right)} \Gamma_{k}(\alpha+\beta t)^{a}}{(1-q)^{b \beta t} \Gamma_{q}(\alpha+\beta t)^{b}}, \quad t \in(0, \infty), k>0, q \in(0,1) .
$$

where $a, b, \alpha, \beta$ are positive real numbers. Then $\Upsilon$ is increasing on $t \in(0, \infty)$ and the inequality

$$
0<\frac{\Gamma_{k}(\alpha+\beta t)^{a}}{\Gamma_{q}(\alpha+\beta t)^{b}}<\frac{e^{a \beta(t-1)\left(\frac{\ln k-\gamma}{k}\right)} \Gamma_{k}(\alpha+\beta)^{a}}{t^{a \beta}(1-q)^{b \beta(1-t)} \Gamma_{q}(\alpha+\beta)^{b}}
$$

holds for every $t \in(0,1)$.

Proof. Let $h(t)=\ln \Upsilon(t)$ for every $t \in(0, \infty)$. Then,

$$
\begin{aligned}
h(t) & =\ln \frac{t^{a \beta} e^{-a \beta t\left(\frac{\ln k-\gamma}{k}\right)} \Gamma_{k}(\alpha+\beta t)^{a}}{(1-q)^{b \beta t} \Gamma_{q}(\alpha+\beta t)^{b}} \\
& =-a \beta t\left(\frac{\ln k-\gamma}{k}\right)-b \beta t \ln (1-q)+a \beta \ln t+a \ln \Gamma_{k}(\alpha+\beta t)-b \ln \Gamma_{q}(\alpha+\beta t)
\end{aligned}
$$

Then,

$$
\begin{aligned}
h^{\prime}(t) & =-a \beta\left(\frac{\ln k-\gamma}{k}\right)-b \beta \ln (1-q)+\frac{a \beta}{t}+a \beta \psi_{k}(\alpha+\beta t)-b \beta \psi_{q}(\alpha+\beta t) \\
& =\beta\left[-a\left(\frac{\ln k-\gamma}{k}\right)-b \ln (1-q)+\frac{a}{t}+a \psi_{k}(\alpha+\beta t)-b \psi_{p}(\alpha+\beta t)\right]>0
\end{aligned}
$$


as a result of Lemma 2.5. This proves that $h$ is increasing on $t \in(0, \infty)$. Hence $\Upsilon$ is increasing on $t \in(0, \infty)$ and for every $t \in(0,1)$ we have

$$
\Upsilon(0)<\Upsilon(t)<\Upsilon(1)
$$

yielding

$$
0<\frac{\Gamma_{k}(\alpha+\beta t)^{a}}{\Gamma_{q}(\alpha+\beta t)^{b}}<\frac{e^{a \beta(t-1)\left(\frac{\ln k-\gamma}{k}\right)} \Gamma_{k}(\alpha+\beta)^{a}}{t^{a \beta}(1-q)^{b \beta(1-t)} \Gamma_{q}(\alpha+\beta)^{b}} .
$$

Corollary 3.4. If $t \in[1, \infty)$, then the following inequality holds.

$$
\frac{e^{a \beta(t-1)\left(\frac{\ln k-\gamma}{k}\right)} \Gamma_{k}(\alpha+\beta)^{a}}{t^{a \beta}(1-q)^{b \beta(1-t)} \Gamma_{q}(\alpha+\beta)^{b}} \leq \frac{\Gamma_{k}(\alpha+\beta t)^{a}}{\Gamma_{q}(\alpha+\beta t)^{b}}
$$

Proof. If $t \in[1, \infty)$, then we have $\Upsilon(1) \leq \Upsilon(t)$ yielding the result.

\section{Concluding Remarks}

Remark 4.1. By putting $a=b=\beta=1$ into inequalities (14) and (16), we thus obtain respectively, inequalities (8) and (9) as in [6].

\section{References}

[1] R. Díaz and E. Pariguan, On hypergeometric functions and Pachhammer k-symbol, Divulgaciones Matemtícas 15(2)(2007), 179-192.

[2] V. Krasniqi, T. Mansour and A. Sh. Shabani, Some Monotonicity Properties and Inequalities for $\Gamma$ and $\zeta$ Functions, Mathematical Communications 15(2)(2010), 365-376.

[3] V. Krasniqi and F. Merovci, Logarithmically completely monotonic functions involving the generalized gamma function, Le Matematiche LXV(2010), 15-23.

[4] T. Mansour, Some inequalities for the q-Gamma Function, J. Ineq. Pure Appl. Math. 9(1)(2008), Art. 18.

[5] F. Merovci, Power Product Inequalities for the $\Gamma_{k}$ Function, Int. Journal of Math. Analysis 4(21)(2010), 1007-1012.

[6] K. Nantomah and M. M. Iddrisu, Some Inequalities Involving the Ratio of Gamma Functions, Int. Journal of Math. Analysis 8(12)(2014), 555-560.

Received: March 19, 2014 